\title{
Collision dynamics of co-propagating solitons in mode-locked fibre laser with net-anomalous dispersion
}

Kudelin, Igor

Igor Kudelin, "Collision dynamics of co-propagating solitons in mode-locked fibre laser with net-anomalous dispersion," Proc. SPIE 11671, Real-time Measurements, Rogue Phenomena, and Single-Shot Applications VI, 116710C (5 March 2021); doi: 10.1117/12.2583144

SPIE. Event: SPIE LASE, 2021, Online Only 


\title{
Collision dynamics of co-propagating solitons in mode-locked fibre laser with net-anomalous dispersion
}

\author{
Igor Kudelin \\ Aston Institute of Photonic Technologies, Aston University, Birmingham, United Kingdom
}

\begin{abstract}
Nowadays, real-time measurements of complex dynamics of ultrashort structures in dissipative nonlinear systems have attracted a lot of interest. In this work, we present experimentally obtained real-time dynamics of two closely-separated solitons in a mode-locked erbium fibre laser. Both solitons experienced strong temporal vibrations due to mutual attractive and repulsive forces, alongside with oscillation in their energies. We experimentally demonstrate the influence of the dispersive waves (DWs) on the dynamics of the soliton complex. Eventually, the pulses experienced a collision dynamics that resulted in the formation of a single soliton at a shifted central wavelength with highly energetic Kelly sidebands and increased background radiation. Our experimentally obtained results prove the theoretical works on soliton interaction and enrich the knowledge on the complexities of ultrashort coherent features and their behaviour in nonlinear systems.
\end{abstract}

Keywords: Mode-locked fibre laser, bound solitons, soliton molecule, Dispersive Fourier Transform, dispersive waves

\section{INTRODUCTION}

The great interest to mode-locked lasers is dedicated by their ability to generate ultrafast coherent structures that are widely used in various scientific fields. ${ }^{1}$ Nowadays, multiple types of ultrashort pulses have been theoretically and experimentally investigated, those characteristics are governed by saturable absorber parameters as much as intracavity parameters, such as nonlinearity, dispersion, gain and losses. The variety of ultrashort structures includes dissipative solitons, ${ }^{2}$ stretched pulse ${ }^{3}$ and similaritons. ${ }^{4}$ Moreover, by experiencing attractive and repulsive forces, solitons can establish bound states, ${ }^{5}$ known as soliton molecules due to their particle-like behaviour. Such soliton complexes inherit lower intensity noises and lower time jitter, ${ }^{6}$ demonstrating a potential for the usage in telecommunication ${ }^{7}$ and medical applications. ${ }^{8}$ However, the complex internal dynamics of co-propagating solitons still is incomprehensible and more experimental studies are required.

Recently emerged time-stretch techniques, e. g. the Dispersive Fourier Transformation (DFT), facilitate the experimental studies of non-repetitive dynamics. A mapping of spectra in the time domain, due to propagation in highly-dispersive medium, allows monitoring spectral profile at the multi-MHz rate. ${ }^{9}$ By applying the DFT technique, it becomes feasible to experimentally observe the build-up dynamics of ultrashort coherent structures in complex nonlinear systems such as mode-locked laser, ${ }^{10}$ including formation of counter-propagating solitons. ${ }^{11}$ Moreover, many ultrafast phenomena have been revealed, including spectral pulsations, ${ }^{12}$ soliton explosion, ${ }^{13}$ internal dynamics of soliton molecule ${ }^{14}$ and formation of unsynchronised resonant dispersion waves. ${ }^{15}$ The experimental observations of such phenomena significantly refined the knowledge of ultrafast behaviour in complex nonlinear systems, that was not available to observe by using traditional optical spectrum analysers.

In this work, we present experimental observations of dynamics of closely separated solitons in a modelocked Erbium fibre laser. Both pulses experienced strong vibration in their relative phase and time separation. Moreover, the energies of the main pulse and of the Kelly sidebands ${ }^{16}$ underwent oscillations with modulation depths of 20 percent and 40 percent, respectively. Our analysis demonstrates that the temporal and the pulse energy oscillations were occurring in-phase, while oscillations of the energy in Kelly sidebands had a phase difference of $\pi / 2$, which helped us to conclude about the influence of the Kelly sidebands on the relative forces

Further author information: (Send correspondence to I.K.)

I.K.: E-mail: kudelini@aston.ac.uk, Telephone: +44 (0)7598 933189

Real-time Measurements, Rogue Phenomena, and Single-Shot Applications VI, edited by

Daniel R. Solli, Georg Herink, Serge Bielawski, Proc. of SPIE Vol. 11671, 116710C

(C) 2021 SPIE · CCC code: $0277-786$ X/21/\$21 · doi: 10.1117/12.2583144

Proc. of SPIE Vol. 11671 116710C-1 
between co-propagating pulses. After all, both solitons collided and a new pulse pulse originated with highlyenergetic Kelly sidebands and with a shift of the central wavelength of $1.22 \mathrm{~nm}$ to the shorter wavelength region. We anticipate that this experimental observation advances the understanding of the soliton-soliton and soliton-dispersive-wave interactions and their behaviour in nonlinear systems in general.

\section{EXPERIMENTAL SETUP}

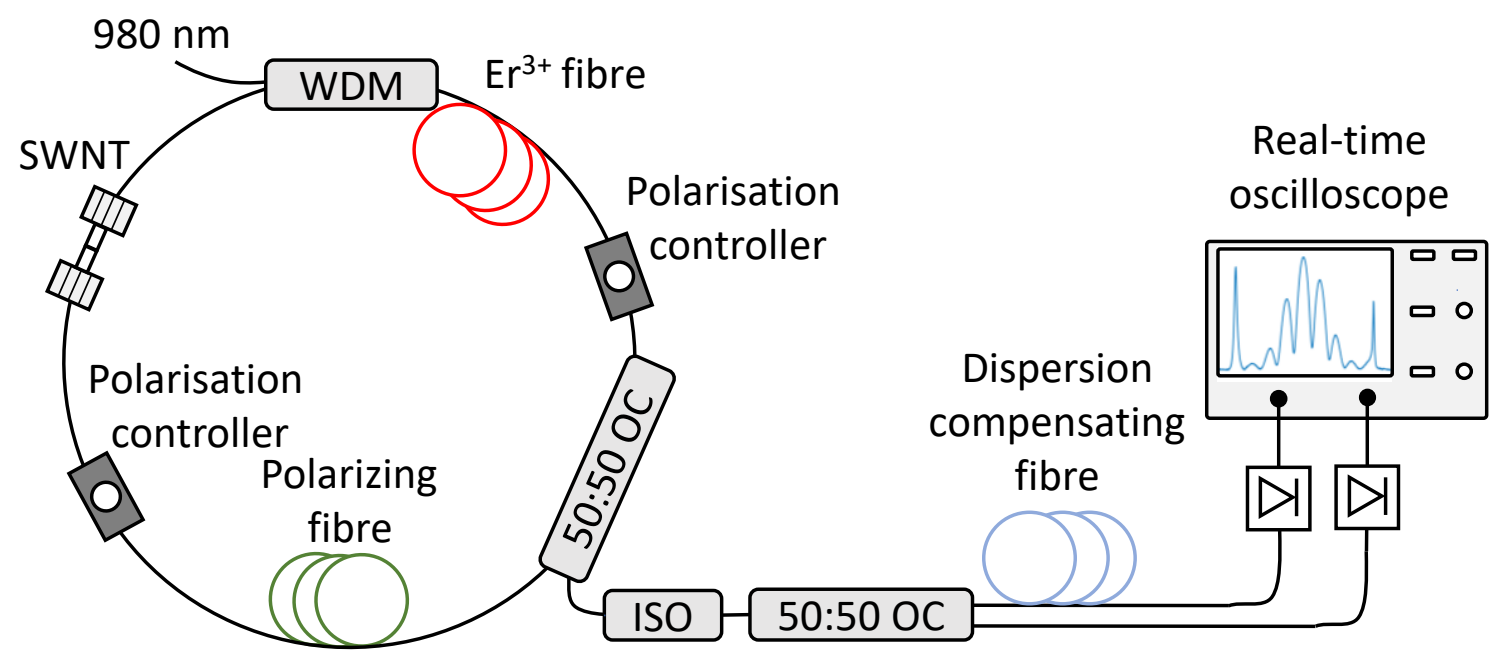

Figure 1. The experimental setup of mode-locked ring fiber laser for DFT measurements. WDM - wavelength division multiplexor, SWNT - single-walled carbon nanotubes, ISO - optical isolator, OC - optical coupler.

Figure 1 presents the measurement setup for the experiment. It consists of a mode-locked fibre laser ${ }^{17}$ and dispersion compensating fibre as a DFT line to implement the real-time measurements. We used $1.8 \mathrm{~m}$ of erbiumdoped fibre (Liekki Er30-4/125) as an active fibre pumped via diode laser at $980 \mathrm{~nm}$ through wavelength division multiplexor. For a stable unidirectional mode-locking generation with robust self-staring, we have used hybrid mode-locking by exploiting both single-walled carbon nanotubes and nonlinear polarisation rotation (NPR). To achieve NPR effect, we have used $6 \mathrm{~m}$ of bent optical polarising fibre (HB1550Z from Thorlabs) with bowtie geometry as a polarisation-selective element that provides the extinction ratio of $30 \mathrm{~dB}$. To adjust a stable unidirectional operation regime, two polarisation controllers (PCs) were used. As a laser output, we used a $3 \mathrm{~dB}$ output coupler. The pulse duration constitutes $570 \mathrm{fs}$ at a repetition rate of $15 \mathrm{MHz}$.

The DFT line consists of $11 \mathrm{~km}$ of dispersion compensating fibre with normal total dispersion of $D=1200 \mathrm{~ns} / \mathrm{nm}$ at $1550 \mathrm{~nm}$. One output line from the last OC passed through the DFT line, while the other has been directly detected on photodiode for spatio-temporal measurements. As measurement equipment, we have used a pair of $50 \mathrm{GHz}$ photodetectors (Finisar) and a $33 \mathrm{GHz}$ real-time storage oscilloscope. The electrical-bandwidth resolution for the DFT measurements has been limited by $0.021 \mathrm{~nm}$.

\section{RESULTS}

Firstly, by adjusting the PCs, we achieved the generation of two spare solitons with separation of a few nanoseconds. Then, with fine control of the PCs, we obtained two loosely bound solitons ${ }^{18}$ with a separation of $\sim 200 \mathrm{ps}$ (the start of our DFT measurements). Finally, by the further tuning of the PCs, the solitons were additionally attracted to each other and their relative distance decreased to less than 15 ps. Under these conditions, both pulses experienced complex vibration-like dynamics that ends up in their collision with the production of a single soliton. The whole spectra dynamics from a loosely bound solitons condition to a new-born pulse is shown in Fig. 2(a). According to a Wiener-Khinchin theorem, we calculated the first-order autocorrelation function ${ }^{19}$ (ACF), depicted in Fig. 2(b). Figure 2(c) shows energy dynamics of the main pulse in blue (excluding Kelly sidebands), background radiation (red line), exclusively Kelly sidebands (orange) and the total intracavity energy 

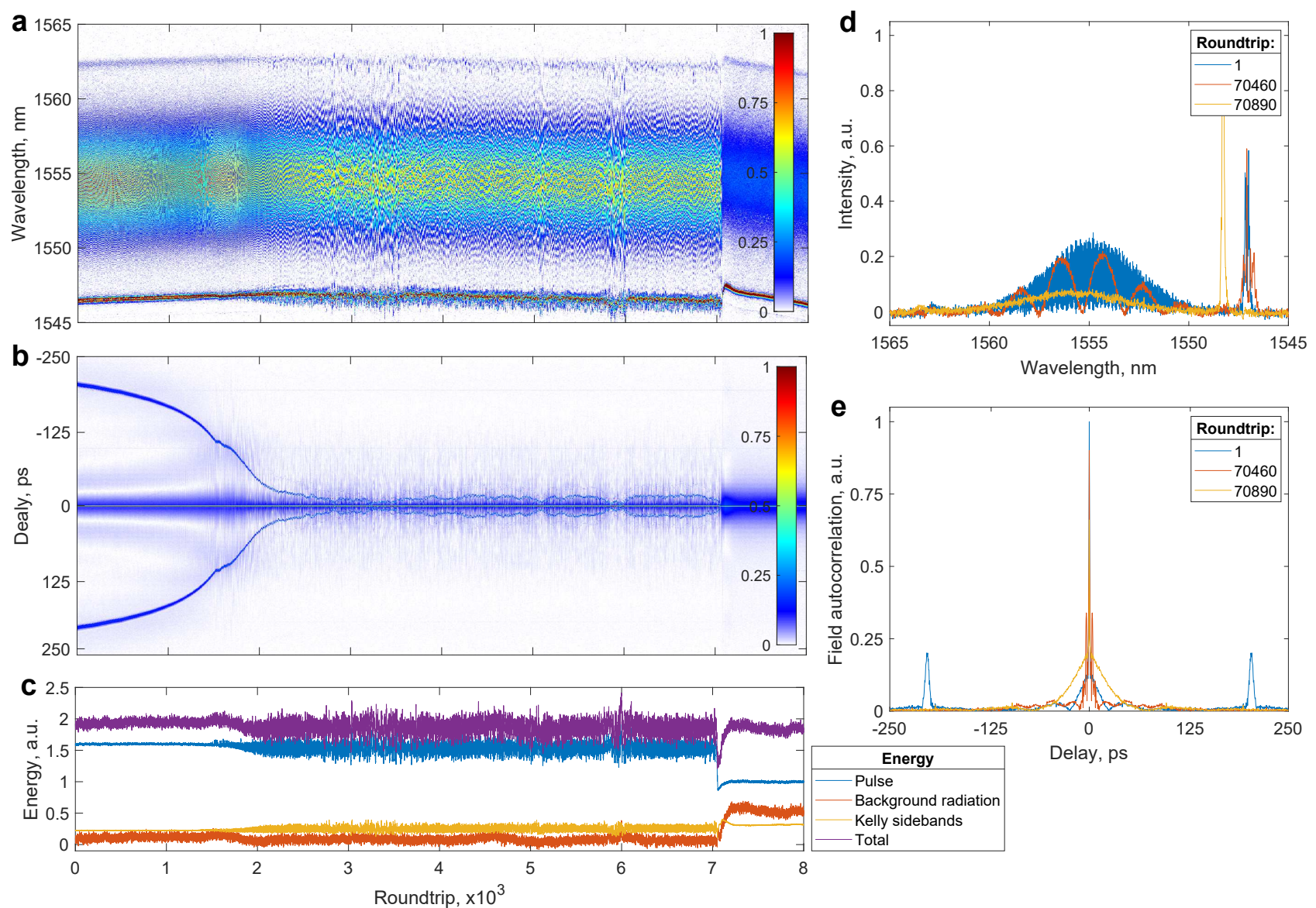

Figure 2. a The DFT spectral dynamics of the pulse colliding with $\mathbf{b}$ corresponding first-order autocorrelation function. c Energy of the pulse without Kelly sidebands, energy in Kelly sidebands and energy of the background radiation. d Cross-section of the DFT pulse spectra and e corresponding first-order autocorrelation function at 1, 70460 (prior pulse collision) and 70890 roundtrips.

in purple. Figures 2(d) and (e) show cross-sections of the DFT measurements and corresponding ACFs for the cases of the loosely bound solitons ( $1^{\text {st }}$ roundtrip), prior pulse colliding and right after (roundtrip 70460 and 70890 respectively). Note, the wings in the ACFs are related to the dispersive waves (Kelly sidebands) that inhibit quasi-continuous wave nature and appear beside the main part of the pulse.

Figure 3 shows a typical vibration-like dynamics of the closely separated solitons in our observations. Note that the dynamics of the Kelly sidebands is analogous to the unsynchronised resonant dispersive wave, recently theoretically described ${ }^{20}$ and experimentally observed with the DFT technique. ${ }^{15}$ Figure 3(c) depicts the energy variations for the pulse excluding Kelly sidebands (blue line) and for Kelly sidebands exclusively (red line). The modulation depths were 20 percent and 40 percent for the pulse energy (excluding Kelly sidebands) and exclusively for Kelly sidebands, respectively. The time separation between the pulses, calculated from the ACF, is presented in Fig. 3(d). The dotted line, shown in Fig. 3(c) and (d), highlights that oscillations of the pulse energy and pulse separation were occurring in-phase, and the closest time separation corresponds to the lower pulses energy. At the same time, the oscillation of the energy in the Kelly sidebands had a $\pi / 2$ shift, that meant that the behaviour of the Kelly sidebands was slightly delayed and caused by the aforementioned changes. In other words, less pulse separation corresponds to a decrease in the energy of the Kelly sidebands, that in turn induces a repulsive force between the pulses. On the other hand, at the points with the largest time separation the attractive force starts to dominate, and the pulses start to converge again. Similar vibration behaviour, governed by the DWs, has been numerically studied in Ref. ${ }^{21}$ Figure 3(e) demonstrates the relative phase between the pulses. The relative phase was retrieved from the DFT spectra with the numerical method described in Ref. ${ }^{22}$ 

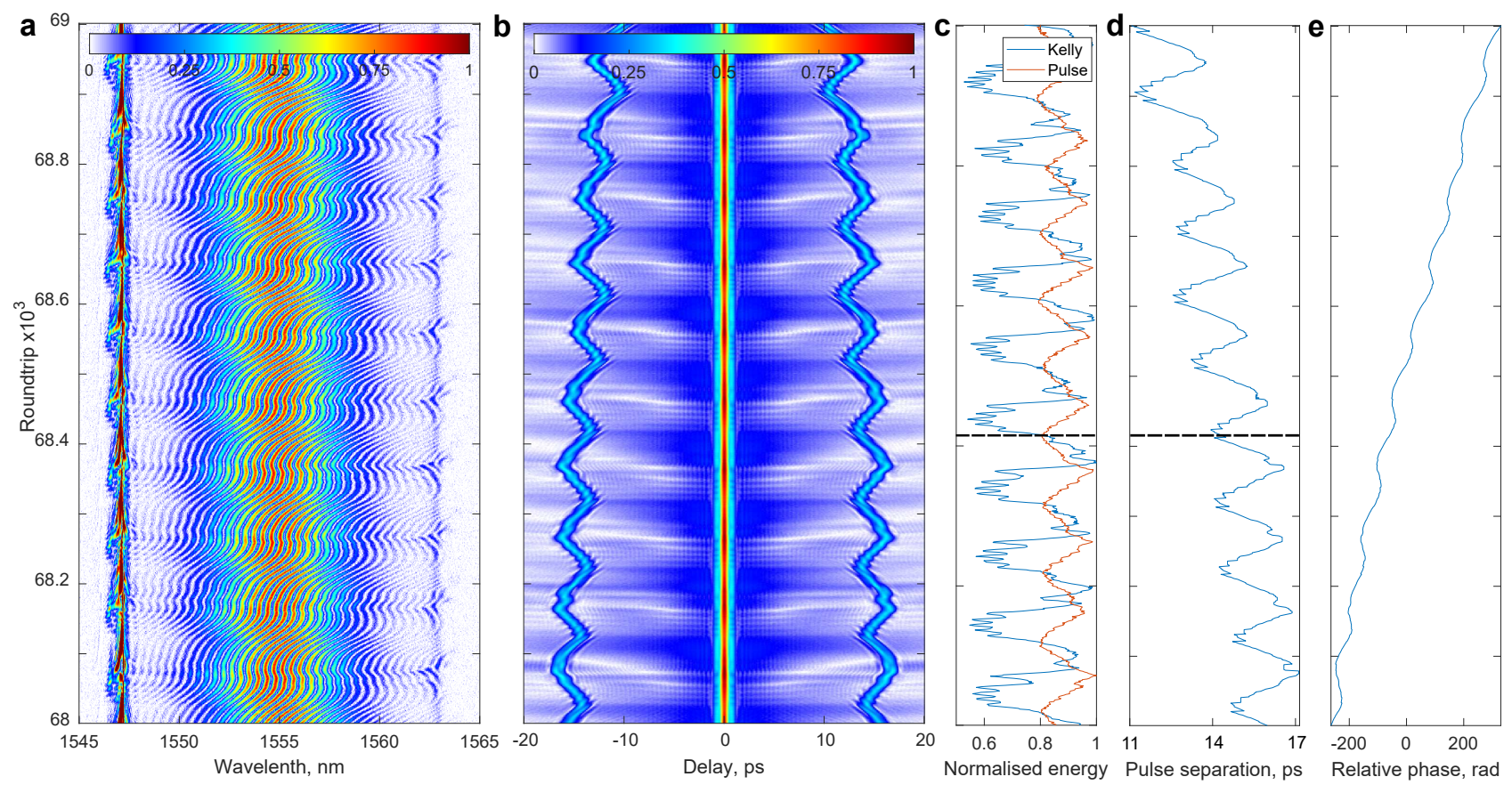

Figure 3. a The DFT spectral dynamics with $\mathbf{b}$ corresponding first-order autocorrelation function. c Normalised energy of the Kelly sidebands and the pulse energy excluding Kelly sidebands. $\mathbf{d}$ Temporal separation between the pulses and $\mathbf{e}$ their relative phase.

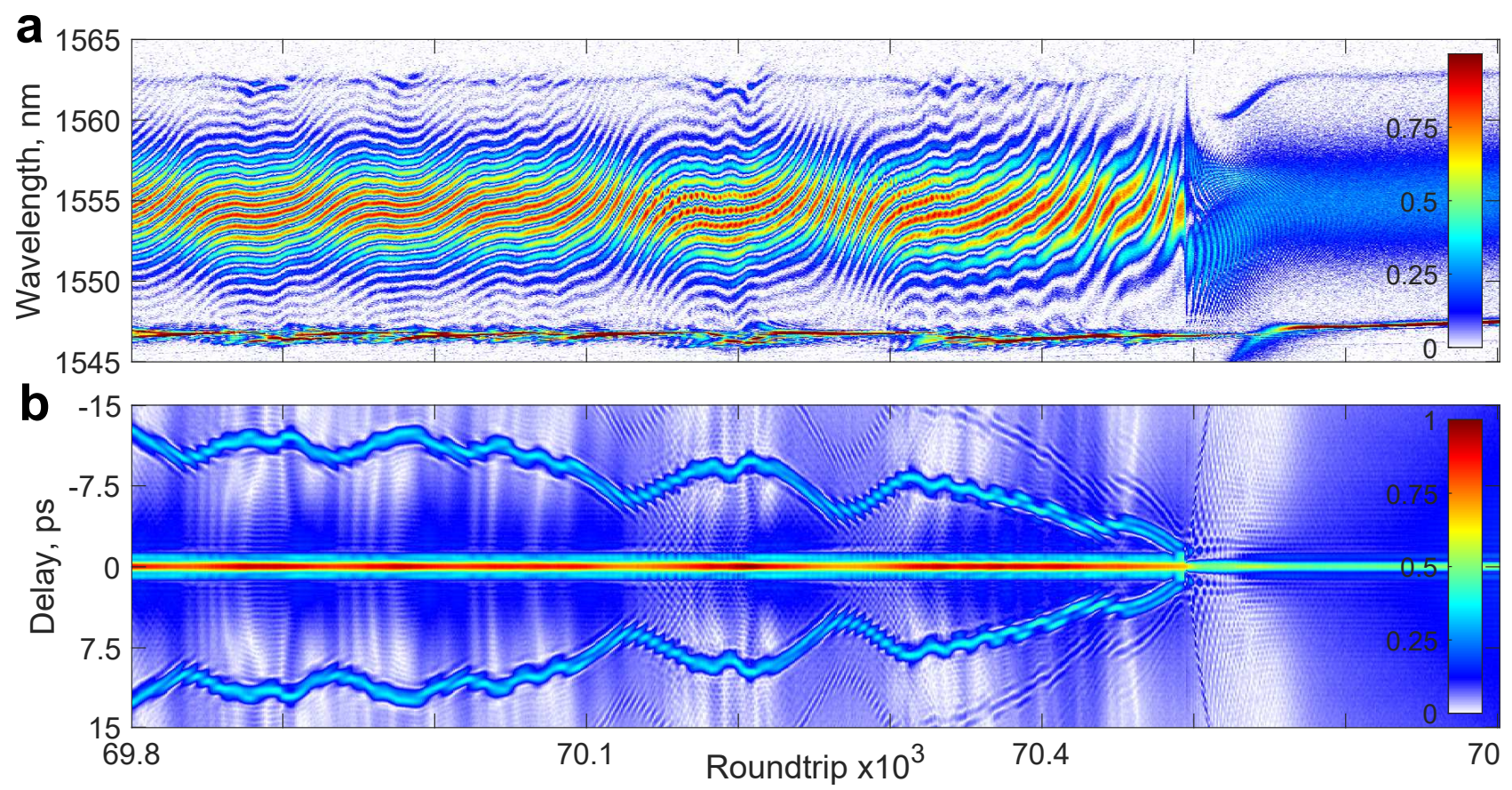

Figure 4. a The DFT spectral dynamics of the pulse colliding with $\mathbf{b}$ corresponding first-order autocorrelation function. 
The relative phase had a strong upward tendency, but at the inflection points with the largest time separation, the phase was locked for $\sim 20$ roundtrips. Nevertheless, throughout the whole dynamics, the phase was rapidly increasing and did not demonstrate a significant role in our observations. This dynamics was observed for four thousand roundtrips.

The final stage of the pulse collision is presented in Fig. 4. Dissimilar to the dynamics shown in Fig. 3, the pulses have a stronger attractive force, which leads to their collision. Since we did not observe noticeable drawdown in the energy of the pulse or DWs, we suppose that the equilibrium between attractive and repulsive forces was disturbed by the PCs adjustment. The DFT spectra show that the pulse collision results in a generation at two distinguished wavelengths at a shorter wavelength of $1551.6 \mathrm{~nm}$ and at a longer wavelength of $1558.1 \mathrm{~nm}$ (roundtrip 70500 in Fig. 4(a)). We suppose that this division of wavelengths was occurred because of strong modulation due to interference at a particular relative phase between the pulses. However, in course of 100 roundtrips, both wavelengths evolve into one solitonic-shape spectrum with a shift in the central wavelength of $1.22 \mathrm{~nm}$ to the shorter wavelength. Strong interference between the pulses and emitted DWs can be also clearly seen from the ACF (Fig. 4(b)) when the pulse separation is less than 10 ps ( 20 times larger than the pulse duration). Due to stronger interference at shorter time distances $(\sim 4 \mathrm{ps})$, the shape of the side pulse resembles a double-spike structure with a dip in the centre (from roundtrip 70400 to 70500 in Fig. 4(b)). Similar to our previous observations with Q-switched instabilities,${ }^{15}$ the newly-generated pulse was accompanied by a significant drop in energy ( $\sim 30$ percent, as shown in Fig. 2$)$ and had a satellite pulse with significantly less energy.

\section{CONCLUSION}

Here, we have presented experimental observations of the complex dynamics of closely-separated solitons. The soliton structure experienced oscillations in their relative time distance and energies of either the pulse and Kelly sidebands. The vibration-like behaviour is explained by the attractive force due to PCs adjustments and the repulsive force, induced by the Kelly sidebands. Moreover, we demonstrated that the relative phase is not affect the vibration dynamics, however, it may affect the central wavelength of the newly-generated pulses. We believe that experimental observations, presented in this paper, will move forward the knowledge and applicability of co-propagated solitons and solitons molecules.

\section{ACKNOWLEDGMENTS}

This unnumbered section is used to identify those who have aided the authors in understanding or accomplishing the work presented and to acknowledge sources of funding.

\section{REFERENCES}

[1] Fermann, M. E. and Hartl, I., "Ultrafast fiber laser technology," IEEE Journal of Selected Topics in Quantum Electronics 15(1), 191-206 (2009).

[2] Grelu, P. and Akhmediev, N., "Dissipative solitons for mode-locked lasers," Nature Photonics 6(2), 84 (2012).

[3] Tamura, K., Ippen, E. P., Haus, H. A., and Nelson, L. E., "77-fs pulse generation from a stretched-pulse mode-locked all-fiber ring laser," Opt. Lett. 18, 1080-1082 (Jul 1993).

[4] Oktem, B., Ülgüdür, C., and Ilday, F. Ö., "Soliton-similariton fibre laser," Nature Photonics 4(5), 307 (2010).

[5] Tang, D., Man, W., Tam, H., and Drummond, P., "Observation of bound states of solitons in a passively mode-locked fiber laser," Physical Review A 64(3), 033814 (2001).

[6] Dvoretskiy, D. A., Sazonkin, S. G., Kudelin, I. S., Orekhov, I. O., Pnev, A. B., Karasik, V. E., and Denisov, L. K., "Multibound soliton formation in an erbium-doped ring laser with a highly nonlinear resonator," IEEE Photonics Technology Letters (2019).

[7] Chernysheva, M., Bednyakova, A., Al Araimi, M., Howe, R. C., Hu, G., Hasan, T., Gambetta, A., Galzerano, G., Rümmeli, M., and Rozhin, A., "Double-wall carbon nanotube hybrid mode-locker in tm-doped fibre laser: A novel mechanism for robust bound-state solitons generation," Scientific reports 7, 44314 (2017). 
[8] Kerse, C., Kalaycığlu, H., Elahi, P., Çetin, B., Kesim, D. K., Akçaalan, Ö., Yavaş, S., Aşık, M. D., Öktem, B., Hoogland, H., et al., "Ablation-cooled material removal with ultrafast bursts of pulses," $N a$ ture 537(7618), 84-88 (2016).

[9] Goda, K. and Jalali, B., "Dispersive fourier transformation for fast continuous single-shot measurements," Nature Photonics 7(2), 102 (2013).

[10] Herink, G., Jalali, B., Ropers, C., and Solli, D., "Resolving the build-up of femtosecond mode-locking with single-shot spectroscopy at $90 \mathrm{mhz}$ frame rate," Nature Photonics 10(5), 321 (2016).

[11] Kudelin, I., Sugavanam, S., and Chernysheva, M., "Build-up dynamics in bidirectional soliton fiber lasers," Photonics Research 8(6), 776-780 (2020).

[12] Peng, J., Boscolo, S., Zhao, Z., and Zeng, H., "Breathing dissipative solitons in mode-locked fiber lasers," Science Advances 5(11) (2019).

[13] Peng, J. and Zeng, H., "Soliton collision induced explosions in a mode-locked fibre laser," Communications Physics 2(1), 34 (2019).

[14] Herink, G., Kurtz, F., Jalali, B., Solli, D., and Ropers, C., "Real-time spectral interferometry probes the internal dynamics of femtosecond soliton molecules," Science 356(6333), 50-54 (2017).

[15] Kudelin, I., Sugavanam, S., and Chernysheva, M., "Pulse-onset dynamics in a bidirectional mode-locked fibre laser via instabilities," Communications Physics 3(1), 1-10 (2020).

[16] Kelly, S., "Characteristic sideband instability of periodically amplified average soliton," Electronics Letters 28(8), 806-807 (1992).

[17] Chernysheva, M., Al Araimi, M., Kbashi, H., Arif, R., Sergeyev, S. V., and Rozhin, A., "Isolator-free switchable uni-and bidirectional hybrid mode-locked erbium-doped fiber laser," Optics Express 24(14), 15721-15729 (2016).

[18] Wu, X., Tang, D., Luan, X., and Zhang, Q., "Bound states of solitons in a fiber laser mode locked with carbon nanotube saturable absorber," Optics Communications 284(14), 3615 - 3618 (2011).

[19] Peng, J., Sorokina, M., Sugavanam, S., Tarasov, N., Churkin, D. V., Turitsyn, S. K., and Zeng, H., "Realtime observation of dissipative soliton formation in nonlinear polarization rotation mode-locked fibre lasers," Communications Physics 1(1), 20 (2018).

[20] Du, Y., Han, M., Cheng, P., and Shu, X., "Pulsating soliton with broadened kelly sidebands in an ultrafast fiber laser," Optics Letters 44(16), 4087-4090 (2019).

[21] Tang, D. Y., Zhao, B., Zhao, L. M., and Tam, H. Y., "Soliton interaction in a fiber ring laser," Phys. Rev. E 72, 016616 (Jul 2005).

[22] Wang, Z., Nithyanandan, K., Coillet, A., Tchofo-Dinda, P., and Grelu, P., "Optical soliton molecular complexes in a passively mode-locked fibre laser," Nature Communications 10(1), 830 (2019). 